\title{
A COMPUTATIONAL STUDY OF THE EFFECT OF EXPERIENCE ON PROBLEM/SOLUTION SPACE EXPLORATION IN TEAMS
}

\author{
Perisic, Marija Majda (1); Martinec, Tomislav (1); Storga, Mario (1,2); Gero, John S (3,4) \\ 1: University of Zagreb; 2: Luleå University of Technology; 3: University of North Carolina at \\ Charlotte; 4: George Mason University
}

\begin{abstract}
This paper presents the results of computational experiments aimed at studying the effect of experience on design teams' exploration of problem-solution space. An agent-based model of a design team was developed and its capability to match theoretically-based predictions is tested. Hypotheses that (1) experienced teams need less time to find a solution and that (2) in comparison to the inexperienced teams, experienced teams spend more time exploring the solution-space than the problem-space, were tested. The results provided support for both of the hypotheses, demonstrating the impact of learning and experience on the exploration patterns in problem and solution space, and verifying the system's capability to produce the reliable results.
\end{abstract}

Keywords: Teamwork, Simulation, Human behaviour in design, Agent-based modelling, Coevolution

\section{Contact:}

Perisic, Marija Majda

University of Zagreb, FSB

Department of Design

Croatia

mperisic@fsb.hr 


\section{INTRODUCTION}

Although many design activities are carried out in a team, factors influencing design team processes and behaviours are still inadequately understood. To support studies of teams, the overarching goal of this research is to develop a computational system capable of simulating various aspects of teamwork in design. Notably, a computational laboratory could provide valuable insights into team properties and behaviours difficult to study in real-world experiments (e.g. change in cognitive behaviour of designers over long periods). To accommodate the capability requirements of such a computational system, a model in which individuals are represented with computational agents was developed and implemented. The agents are based on social and cognitive theories and are capable of learning, thus providing them with the capability to change over time, and offering a means to simulate and study the adaptation processes of design teams.

In the previous work (Perišić et al., 2017; 2019), the computational model was designed, implemented, and tested for its ability to capture the trends observed in the real world. Namely, the focus of previous studies was on the effect of experience and learning on the behaviour of stable teams. The studies tested whether the agents upon being faced with a task for the second time, are capable of solving it quicker and with less effort in searching the knowledge space.

To further refine an understanding of team behaviour using this computational system, this paper focuses on the effect of experience on agents' progress in problem-solution space in more detail.

Through testing of two theoretically-based hypotheses, this study is aimed at assessing the capability of the computational system to capture the empirically found differences in problem-solution space exploration trends of inexperienced and experienced designers (i.e. verification), and also at offering additional insights into the behaviour of design teams.

\section{THEORETICAL BACKGROUND}

Studies have shown that expertise affects designers' effectiveness in performing design-related tasks, particularly that experts tend to outperform novice designers (Sun et al., 2016; Cross, 2004). Petre (2004) summarised the literature on design expertise into several characteristics of experienced designers, including that they store information in larger and organised chunks and that they remember a large number of solution examples. Hence, experts might find it easier to directly propose solutions to particular sub-problems (Liikkanen and Perttula, 2009). Ahmed et al. (2003) pointed out that novice designers demonstrate the iterative pattern of trial and error, whereas experienced designers adopt particular strategies to address the given problem more efficiently. In their study of expert and novice designers, Kavakli and Gero (2002) describe experts as cognitively more active and productive, and their cognitive actions as better organised and structured. McComb et al. (2015) have shown that the individuals in high performing teams behave as experts in a sense that they quickly select a good direction in which to search for a solution and maintain a low level of divergence. Consequently, experts are likely to consider less options than novices, in favour of immediately producing higher quality solutions (Björklund, 2013).

Cross (2001) found that designers who differ in experience are likely to exhibit different proportions of time spent in the problem and solution spaces. It is suggested that inexperienced designers spend more time defining the problem, whereas designers with specific experience of the problem type tend to approach the task through solution conjectures (Cross, 2001; Lloyd and Scott, 1994). Cross (2004) attributed such behaviour to the experts' exposure to a larger number of examples of the problems and solutions in a particular domain. He concluded that due to this exposure, experts are "able to store and access information in larger cognitive 'chunks' than novices can, and to recognise underlying principles, rather than focussing on the surface features of problems". Liikkanen and Perttula (2009) support these findings by demonstrating how novice designers start by focusing on the problem structure if they cannot directly recognise a satisfactory solution, while experts recall more complex solutions without performing extensive decomposition of the given design problem.

\section{AIM AND HYPOTHESES}

Building on this theoretical foundation, the current work aims to create a computational model of a design team which will be used to test the following two hypotheses: 
H1: Experienced (expert) teams perform better than inexperienced (novice) teams in terms of time needed to find a solution.

H2: Experienced teams spend more time than inexperienced teams exploring the solution space than the problem space.

\section{MODEL}

The agent-based computational model comprises cognitively rich, social agents (Perišić et al., 2019). To describe agents' mental models, the agents' knowledge space is modelled as a directed, threelayered network where each layer corresponds to a domain from the Function-Behaviour-Structure ontology (Gero, 1990), and contains nodes of its respective type (i.e. nodes representing functions, behaviours or structures). Directed links connect function nodes to behaviour nodes, and behaviour nodes to structure nodes. There are no direct links between nodes of the same type. Further, structure nodes can be reached from function nodes only through the behaviour nodes (Gero and Kannengiesser, 2004).

To enable reasoning about the validity of links within the knowledge space, each structure node is associated with a network, Figure 1, while each behaviour node represents an interval of values of one of the following network metrics: average degree centrality, average closeness centrality, average betweenness centrality, diameter and clustering coefficient. Behaviour is derived from a structure by computing these metrics on the network associated with the structure. In this manner, an association between behaviour and structure nodes is created.

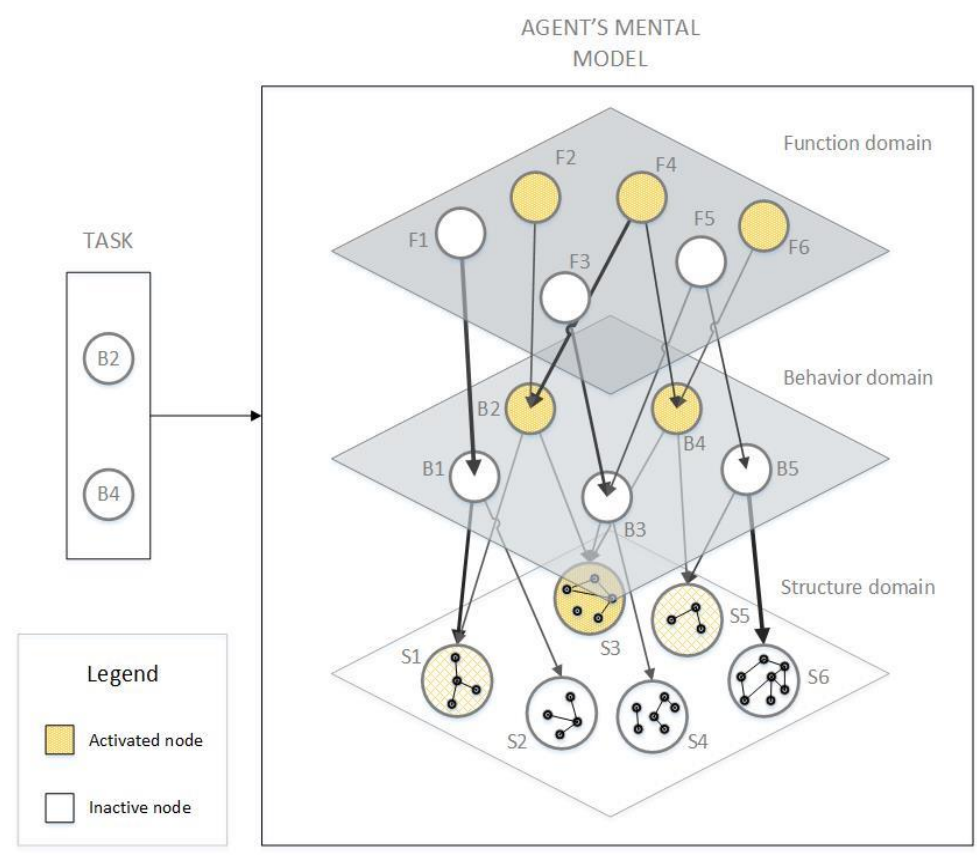

Figure 1. Agent's mental model (Perišić et al., 2019)

Each agent's mental model consists of a portion of the knowledge space, and - as depicted in Figure 1 the agent's reasoning processes are modelled as an activation spreading over its mental model (Perišić et al., 2019). To indicate the associative relation between knowledge nodes (i.e. function, behaviour and structure nodes), the knowledge links within the agent's mental model are weighted. The weights of the knowledge links change by their usage, i.e., they become more grounded. As the link is being used (i.e. activation is spread along it), its weight increases, and it becomes easier to process. This results in a change of the amount of time required to pass the activation over the link. If the link weight exceeds a specified threshold, the link becomes more grounded, and the agent can process it at much lower cost, thus enabling the activation to reach nodes which are two or more links apart in a single step. This mode of reasoning corresponds to System 1 thinking in the dual process theory of cognition (Kahneman, 2011), or reflexive reasoning (Maher and Gero, 2002). However, if the link is not used, the agent slowly forgets its utility by decreasing the link's weight. 
At the simulation start, agents are created and primed with their expertise area, which is implemented as a portion of well-grounded knowledge. A set of tasks is generated so each task is defined as a set of requirements which a structure (solution) is required to meet to be accepted as a solution. When agents are presented with a task, the initial activation is generated and agents attempt to pass it to function nodes, and further through behaviour to structure nodes. Thus, upon seeing the task, agents have to determine which function nodes are relevant. Passing the activation from requirement to function nodes and further to behaviour nodes, corresponds to the process of Formulation in the FBS ontology (Gero and Kannengiesser, 2004). While passing of activation from behaviour to structure nodes corresponds to the process of Synthesis. Once a structure node is sufficiently activated, the agent can analyse it by computing the associated network's properties (Analysis), and then evaluate it by comparing the expected and obtained behaviours, i.e. network property values (Evaluation). Through the success of such processes, agents can learn new links between the knowledge nodes.

If the agent determines that the structure is unsatisfactory (through Evaluation), it can direct its attention to the unmet requirements by passing the activation to the relevant functions (Reformulation III in the FBS ontology). Finally, agents can expand the solution space by combining the respective networks of two sufficiently active structure nodes (Reformulation I in the FBS ontology). In such a way, new networks - and, consequently, new structure nodes - are created. The newly created networks have different properties than the subnetworks they were created from, therefore enabling the new structure to display a previously unseen combination of behaviours.

In the computational system, two processes in the FBS ontology, Documentation and Reformulation II, are not modelled. As agents are working in a team, they may decide to share their ideas with others. If an agent perceives a structure to be satisfactory (i.e. a solution), it can propose it to others who evaluate it and report their evaluations. Similarly, if an agent's mental model contains two active nodes connected with a sufficiently grounded link, the agent can decide to communicate the link to others. In one simulation step, only one message can be shared. If more than one agent has a link or a solution to share, the speaker is chosen randomly. Upon hearing the communicated link, other agents either learn it or further ground it, and activate the relevant nodes. The increase in communicated link's weight, as well as the amount of activation sent to the respective nodes, depends on the perceived expertise of the speaking agent (i.e. trust). Trust also plays a role in the acceptance of solutions: agent's evaluations of the proposed structures are weighted by the average trust others held in the agent. Simulation is over when the weighted sum of agents' evaluations of the proposed structure exceeds a predefined threshold.

Agents and tasks are initialised at the simulation start. Agent's personal factors and knowledge network are created. Personal factors influence sharing behaviour, the acceptance of new ideas, and cognitive ability guiding the number of elements which can be processed in a single time step. Further, agents' expertise areas are selected and used to set initial trust among agents. Once agents are given the task, the mechanisms described earlier guide their reasoning processes and communication. Agents possess constrained working memory, enabling them to memorise sufficiently active structures and links. At any time step, the links or structures stored in the working memory can be shared, but the contents of working memory decay over time or change due to new links or structures being activated. When agents agree that the proposed structure meets the requirements, the task is finished. If simulating sequences of tasks, in between two tasks, agents update trust in each other based on the success of the past task. Otherwise, their mental models and trust values are restarted.

\section{DESIGN OF THE EXPERIMENTS}

To test the two hypotheses, the agent-based model was run on a set of 250 computational experiments. Each experiment consisted of four tasks performed by teams of agents. The performance on the initial task was not included in the results and statistics, as it served an "expert" agent team gaining experience which would distinguish it from an inexperienced, "novice" team of agents. The remaining three tasks were then executed by both expert agents and novices. Statistics on the number of steps needed to find a solution, the number of new links learned throughout the task, the number of new structures created throughout the task, and the number of distinct links and structures communicated were collected for both teams. Further, the communication among agents was recorded for each task, which provided a means to study the progress in problem-solution space over time for both team types. 
The novices' mental models were restarted at the beginning of each task, while experienced agents learned over the course of the experiment, i.e., the novices' performance serves to show how a certain team performs when team members have no previous experience, Figure 2.

The first tasks, T1, were chosen to be related to the teams' expertise: each agent's expertise is required to solve the task, but none of the agents is capable of solving it alone. Each subsequent task is chosen to be progressively more difficult than the previous one, in a sense that more requirements need to be satisfied and/or requirements are narrower in scope (i.e. the required behaviours are associated with more restricted values of network metrics).

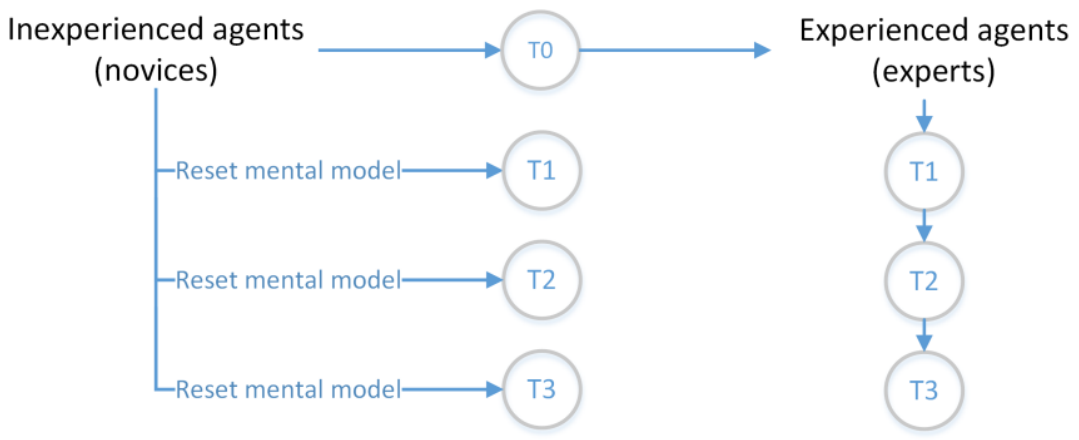

Figure 2. Schematic representation of the experiments

For each experiment, the initial simulation parameters were as follows to provide a range of cases to be explored: the number of initial structure nodes known by one agent was set to 50, the number of function domain nodes was set to 35, and the number of behaviour domain nodes ranged from 15 to 50 (i.e. for each of the five network metrics, at least three, and no more than ten behaviour nodes were created; each node was associated with different ranges of metric values). The number of agents within a team was set to three, as the scalability tests of the computational model demonstrated its capability of producing systematic behaviour even on teams of such size. There was no limit on the number of simulation steps, i.e. the simulations were carried out until the solution was found and accepted by the team.

\section{RESULTS}

\subsection{Summary statistics}

To enable performance evaluations and to enable comparisons with previous work (Perišić et al., 2019), the statistics regarding the number of steps needed to find a solution, the overall number of new links and structures created, as well as the number of distinct links and structures communicated by the team during each task are collected, Table 1. Following the first hypothesis (H1) and the results from previous work, it is expected that experts would require significantly fewer steps than novices to find a satisfying solution. Similarly, it is anticipated that the numbers of structures and knowledge links learned and communicated will be significantly lower for the expert teams. This follows from the expectation that the number of communicated links and structures is bounded by the number of simulation steps, and the same applies for the number of new structures and knowledge links created. Table 1 presents the significance levels obtained while testing for the differences in the distributions of the measures between novices and experts. The Wilcoxon's signed-ranked test was used to perform the analysis.

The results of the simulations show that there are strong significant differences in steps needed by experts and novices to reach a solution, with the experts taking noticeably fewer steps. This provides support for hypothesis $\mathrm{H} 1$.

From the second hypothesis (H2) follows an expectation that experienced teams would concentrate more on the solution space exploration than novices, i.e., experts would typically create and communicate more structures per time step. Since each new structure can be associated with new links (from behaviour nodes to the structure), it is expected that experts will create more links per time step than novices. The mean values and standard deviations on the collected metrics, along with the significance levels of the Wilcoxon's singed-ranked test for the differences between novices and 
experts are presented in Table 2. Related hypotheses were formulated to state that experts would have created and shared more structures and knowledge links per simulation time step than novices.

Table 1. Mean values and standard deviations (in brackets) of measures for each task group

\begin{tabular}{|c|c|c|c|c|c|c|}
\hline & \multicolumn{2}{|c|}{ First task } & \multicolumn{2}{|c|}{ Second task } & \multicolumn{2}{|c|}{ Third task } \\
\hline & Experts & Novices & Experts & Novices & Experts & Novices \\
\hline Steps & $\begin{array}{c}290.7 \\
(381.6)\end{array}$ & $\begin{array}{c}379.59 \\
(401.75)\end{array}$ & $\begin{array}{c}310.56 \\
(451.23)\end{array}$ & $\begin{array}{c}401.49 \\
(436.25)\end{array}$ & $\begin{array}{c}316.24 \\
(421.79)\end{array}$ & $\begin{array}{c}494.7 \\
(452.35)\end{array}$ \\
\hline $\mathrm{p}$ - values & \multicolumn{2}{|c|}{$0.001^{*}$} & \multicolumn{2}{|c|}{$0.000^{*}$} & \multicolumn{2}{|c|}{$0.001 *$} \\
\hline $\begin{array}{l}\text { New } \\
\text { links }\end{array}$ & $\begin{array}{c}125.79 \\
(194.88)\end{array}$ & $\begin{array}{c}135.73 \\
(151.12)\end{array}$ & $\begin{array}{l}144.21 \\
(227.0)\end{array}$ & $\begin{array}{c}126.08 \\
(123.71)\end{array}$ & $\begin{array}{l}201.46 \\
(283.5)\end{array}$ & $\begin{array}{l}172.44 \\
(200.2)\end{array}$ \\
\hline$p$ - values & \multicolumn{2}{|c|}{$0.001 *$} & \multicolumn{2}{|c|}{0.178} & \multicolumn{2}{|c|}{0.7686} \\
\hline $\begin{array}{c}\text { New } \\
\text { structures }\end{array}$ & $\begin{array}{c}26.71 \\
(51.81)\end{array}$ & $\begin{array}{c}28.36 \\
(50.39)\end{array}$ & $\begin{array}{c}34.63 \\
(65.39)\end{array}$ & $\begin{array}{c}26.11 \\
(34.61)\end{array}$ & $\begin{array}{c}57.82 \\
(90.00)\end{array}$ & $\begin{array}{c}37.26 \\
(52.52)\end{array}$ \\
\hline $\mathrm{p}$ - values & \multicolumn{2}{|c|}{$0.039 *$} & \multicolumn{2}{|c|}{0.972} & \multicolumn{2}{|c|}{0.134} \\
\hline $\begin{array}{l}\text { Communicated } \\
\text { links }\end{array}$ & $\begin{array}{c}29.51 \\
(24.44)\end{array}$ & $\begin{array}{c}40.00 \\
(16.82)\end{array}$ & $\begin{array}{c}29.33 \\
(29.63)\end{array}$ & $\begin{array}{l}41.06 \\
(16.8)\end{array}$ & $\begin{array}{c}31.95 \\
(32.02)\end{array}$ & $\begin{array}{l}47.09 \\
(20.89)\end{array}$ \\
\hline$p$ - values & \multicolumn{2}{|c|}{$0.000^{*}$} & \multicolumn{2}{|c|}{$0.000 *$} & \multicolumn{2}{|c|}{$0.000 *$} \\
\hline $\begin{array}{c}\text { Mentioned } \\
\text { structures }\end{array}$ & $\begin{array}{c}26.07 \\
(27.11)\end{array}$ & $\begin{array}{c}30.68 \\
(19.48)\end{array}$ & $\begin{array}{c}27.18 \\
(33.56)\end{array}$ & $\begin{array}{c}29.96 \\
(17.66)\end{array}$ & $\begin{array}{c}34.26 \\
(37.22)\end{array}$ & $\begin{array}{l}38.06 \\
(27.87)\end{array}$ \\
\hline $\mathrm{p}$ - values & \multicolumn{2}{|c|}{$0.000 *$} & \multicolumn{2}{|c|}{$0.001 *$} & \multicolumn{2}{|c|}{0.155} \\
\hline
\end{tabular}

$* \mathrm{p}<0.05$

Table 2. Mean values and standard deviations (in brackets) of measures for each task group - relative to the number of steps needed to finish the task

\begin{tabular}{|c|c|c|c|c|c|c|}
\hline & \multicolumn{2}{|c|}{ First task } & \multicolumn{2}{|c|}{ Second task } & \multicolumn{2}{|c|}{ Third task } \\
\hline & Experts & Novices & Experts & Novices & Experts & Novices \\
\hline $\begin{array}{l}\text { New } \\
\text { links }\end{array}$ & $\begin{array}{c}1.07 \\
(1.05)\end{array}$ & $\begin{array}{c}0.55 \\
(0.37)\end{array}$ & $\begin{array}{l}1.19 \\
(0.97)\end{array}$ & $\begin{array}{l}0.55 \\
(0.4)\end{array}$ & $\begin{array}{c}1.32 \\
(1.02)\end{array}$ & $\begin{array}{c}0.47 \\
(0.30)\end{array}$ \\
\hline $\mathrm{p}$ - values & \multicolumn{2}{|c|}{$0.000 *$} & \multicolumn{2}{|c|}{$0.000 *$} & \multicolumn{2}{|c|}{$0.000 *$} \\
\hline $\begin{array}{c}\text { New } \\
\text { structures }\end{array}$ & $\begin{array}{c}0.14 \\
(0.16)\end{array}$ & $\begin{array}{c}0.09 \\
(0.08)\end{array}$ & $\begin{array}{c}0.17 \\
(0.16)\end{array}$ & $\begin{array}{c}0.08 \\
(0.08)\end{array}$ & $\begin{array}{l}0.26 \\
(0.27)\end{array}$ & $\begin{array}{c}0.09 \\
(0.08)\end{array}$ \\
\hline $\mathrm{p}$ - values & \multicolumn{2}{|c|}{$0.000 *$} & \multicolumn{2}{|c|}{$0.000 *$} & \multicolumn{2}{|c|}{$0.000 *$} \\
\hline $\begin{array}{l}\text { Communicated } \\
\text { links }\end{array}$ & $\begin{array}{c}0.22 \\
(0.14)\end{array}$ & $\begin{array}{c}0.21 \\
(0.15)\end{array}$ & $\begin{array}{c}0.22 \\
(0.15)\end{array}$ & $\begin{array}{c}0.22 \\
(0.16)\end{array}$ & $\begin{array}{c}0.21 \\
(0.14)\end{array}$ & $\begin{array}{c}0.17 \\
(0.12)\end{array}$ \\
\hline $\mathrm{p}$ - values & \multicolumn{2}{|c|}{0.465} & \multicolumn{2}{|c|}{0.779} & \multicolumn{2}{|c|}{$0.038 *$} \\
\hline $\begin{array}{c}\text { Mentioned } \\
\text { structures }\end{array}$ & $\begin{array}{c}0.20 \\
(0.13)\end{array}$ & $\begin{array}{c}0.15 \\
(0.10)\end{array}$ & $\begin{array}{c}0.21 \\
(0.14)\end{array}$ & $\begin{array}{l}0.16 \\
(0.12)\end{array}$ & $\begin{array}{c}0.21 \\
(0.13)\end{array}$ & $\begin{array}{c}0.13 \\
(0.08)\end{array}$ \\
\hline$p$ - values & \multicolumn{2}{|c|}{$0.000 *$} & \multicolumn{2}{|c|}{$0.000 *$} & \multicolumn{2}{|c|}{$0.000 *$} \\
\hline
\end{tabular}

$* \mathrm{p}<0.05$

\subsection{P-S indexes and moving P-S indicators}

To study agent's exploration of problem and solution space in greater depth, the team's communication during the tasks was recorded and analysed. Following the classification of FBS processes proposed in (Gero et al., 2013), if the agent communicated either a link between a requirement and a function node or a link from a function node to a behaviour node, the message was encoded as a problem space exploration. On the other hand, if an agent communicated a link from a behaviour node to a structure node, an agent proposed a structure as a final solution, or team members shared their evaluations on the proposed solution, the message was encoded as a solution space exploration. Such encoding of simulated experiments enable calculations of P-S indexes - the ratio of the number of messages related to the 
problem space to those related to the solution space. If the P-S index has values below 1, the focus of design activity was on the solutions, while values above 1 indicate a problem-focused activity. Similar results can be deduced from the P-S indicator, a value obtained by subtracting the number of solutionfocused time steps from the number of problem-focused time steps, and dividing it with an overall number of time steps resulting in values between +1 and -1 (Milovanovic and Gero, 2018). The more negative the P-S indicator is, the more solution-focused was the activity. The average values of P-S indexes and P-S indicators are presented in Table 3. Following the second hypothesis (H2), it is expected that both, P-S index and P-S indicator will be lower for tasks performed by experts than for those performed by novices. The pairwise difference between distributions was tested using the Wilcoxon's signed-ranked test, and the alternative hypothesis was chosen to state that experts will have lower P-S indexes and P-S indicators. The significance level obtained are presented in Table 3.

Table 3. Average P-S indexes and P-S indicators for each task group

\begin{tabular}{|c|c|c|c|c|c|c|}
\hline & \multicolumn{2}{|c|}{ First task } & \multicolumn{2}{c|}{ Second task } & \multicolumn{2}{c|}{ Third task } \\
\hline & Experts & Novices & Experts & Novices & Experts & $\begin{array}{c}\text { Novice } \\
\mathrm{s}\end{array}$ \\
\hline PS index & 0.132 & 0.145 & 0.148 & 0.136 & 0.153 & 0.145 \\
\hline $\mathrm{p}$ - values & \multicolumn{2}{|c|}{$0.001^{*}$} & \multicolumn{2}{c|}{$0.025^{*}$} & \multicolumn{2}{c|}{0.318} \\
\hline PS indicator & -0.801 & -0.764 & -0.787 & -0.773 & -0.78 & -0.76 \\
\hline $\mathrm{p}$ - values & \multicolumn{2}{|c|}{$0.000^{*}$} & \multicolumn{2}{c|}{$0.010^{*}$} & \multicolumn{2}{c|}{0.264} \\
\hline
\end{tabular}

$* \mathrm{p}<0.05$

In addition to the overall P-S indicators, their temporal variant, the moving average P-S indicators, were calculated. A moving window the size of one-tenth of the simulated task length was selected and local P-S indicators were calculated. Thus, for each simulated task, lists of moving P-S indicators were obtained for both novices and experts. To represent the aggregate values for novices and experts, the moving P-S indicator lists were averaged over each time step, and the results are presented in Figure 3.
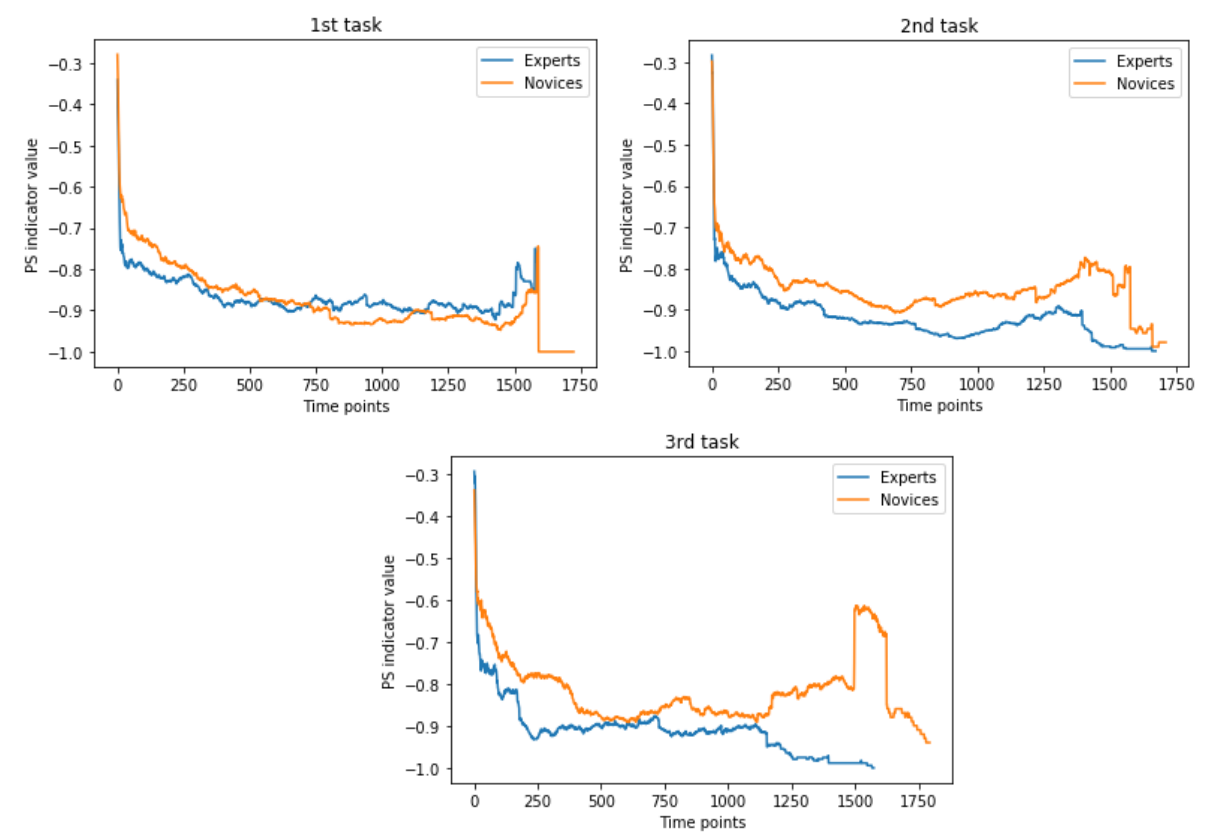

Figure 3. Comparison of the average moving PS indicator values

\subsection{Distributions of design processes}

The final set of results concerns the distributions of FBS processes within the simulated design activities. Due to the implementation characteristics, the numbers of Reformulation I and Evaluation processes communicated by agents are the same: each time an agent proposes a new structure (as a final solution), the agents evaluate it. If an agent intends to mention a new structure but is not certain of its suitability as a solution, it will share a behaviour-structure link. Since there are no direct links between the nodes of the same domain, the agents cannot express Reformulation I in any situation 
other than proposing the final solution. Due to these limitations, the distribution of FBS processes will likely not match the results of the empirical studies. However, observing the averaged distributions of FBS processes can provide additional insight into the agent's behaviour regarding the problemsolution space exploration. These results are presented in Figure 4.

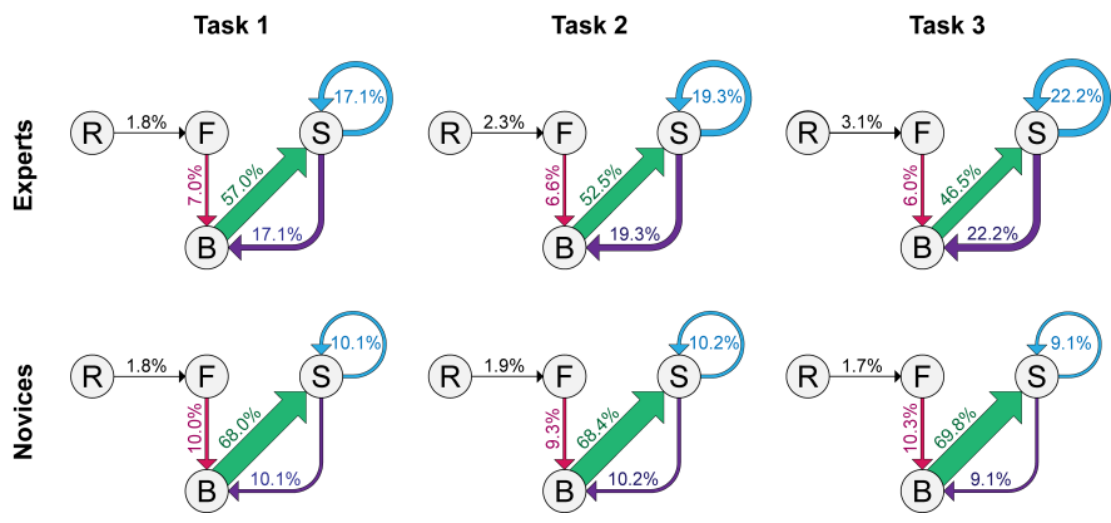

Figure 4. Average distributions of FBS processes for each task group

\section{Discussion}

\subsection{Support for hypothesis 1}

The results presented in Table 1 serve to show that, when compared to less experienced agents, expert agents need fewer steps to find and agree on a structure satisfying the requirements - thus providing support for the first hypothesis: Experienced teams perform better than inexperienced or novice teams in terms of time needed to find a solution. As the difficulty of the tasks increases, for both, novices and experts the average number of steps needed to find a solution increases from task T1 to task T3. However, it can be observed that the rate at which a number of steps increases is lower for the experienced agents, indicating that previous experience provided them with knowledge which is reused in subsequent tasks, while novice agents have to spend additional time learning it. These results are in accordance with the literature (Petre, 2004; Cross, 2004), and provide support for hypothesis H1.

\subsection{Support for hypothesis 2}

As expected, the average absolute number of distinct links communicated by experts is lower than by novices in every task. However, an interesting observation can be made in tasks T2 and T3: while novices spent more time steps solving a task, the absolute number of both, new structures and new knowledge links learnt did not significantly differ between novices and experts. These results match findings from literature (Cross, 2001; Lloyd and Scott, 1994) as they indicate that novices spent time learning the links related to functions and behaviours, while experts were able to transfer to the solution space more quickly and explore it by producing and analysing a larger number of structures. Further, while expert agents analyse non-redundant knowledge nodes and links, the novices spend time reassuring the pre-existing knowledge, which results in a relatively low number of distinct new knowledge links and structures learnt. In task T3, there is no statistically significant difference in the absolute number of structures mentioned by novices and experts. While this again signifies that experts spent more time exploring the solution space, it contradicts the findings that experts consider fewer options than novices (Björklund, 2013). Inspection of the data revealed that, when faced with the task, expert agents quickly activate several relevant structure nodes known from the previous tasks, and tend to suggest them as their solutions, i.e. propose them as solutions for sub-problems (Liikkannen and Perttula, 2009). Inexperienced agents do not know any suitable structures, search for them during the task, and tend to stick to a structure if it is deemed as relevant.

These insights are based on the data in Table 2. While the rate at which expert agents communicate links and structures remains mostly the same, the rate at which they create new structures almost doubles throughout the tasks. This indicates the increased ability to transfer to (and explore) the solution space. As the tasks become more difficult, the inexperienced agents communicate less distinct structures and links, which signifies that knowledge nodes become more difficult to activate and knowledge links take more time steps to ground. These results provide support for hypothesis H2. 


\subsection{More nuanced analysis}

While these results seem to indicate that experts spent more time in the solution space than novices, the P-S indexes presented in Table 3 appear to contradict such findings. Particularly, while for the first two tasks pairwise statistical test determined that the experts' P-S index is significantly lower than the one obtained by the novices, the results for the third task show no statistical difference. Additionally, the average P-S index is greater for the experienced than inexperienced agents. Similarly, the pairwise statistical test of differences in P-S indicators failed to support a significant difference in novices' and experts' performance on tasks T3. The inspection of the data revealed that such results are a consequence of outliers. Namely, as the agents gain experience, the percentage of tasks solved in a very small number of steps (i.e. $<=10$ ) increases. Such tasks are characterised by a short exploration of a problem space (to accommodate for the task requirements) and transfer to a solution space where a satisfying solution is quickly found, proposed and accepted. In other words, these tasks have a higher (but small) number of steps spent in problem space, than in a solution space, and therefore have a comparatively large P-S index. Acting as outliers, these tasks skew the average P-S index and P-S indicator values.

These outliers are the reason why the plots presented in Figure 3 convey such a different message than data shown in Table 3. When averaging the P-S indicators over every time step, short sessions with high $\mathrm{P}-\mathrm{S}$ indicators influence only the first few time step values, which have high P-S indicators in general, as teams are starting the task by exploring the problem space. The remaining (longer) tasks help represent the overall trends in the tasks. Although Tables 1, 2 and 3 offer evidence that in general experts solve tasks T1 quicker and spend more time in a solution than in problem space, Figure 3 shows that there are still tasks in which experts are unable to find solutions quickly - on such tasks their behaviour does not differ much from those of novices. However, as they gain more experience, experts start differing from novices even on the tasks for which they were unable to find a solution for a longer period. While the novices' average moving P-S indicator remains close to -0.8 , the expert's lowers to values around -0.9 . Further, there are increases in the average moving P-S indicators towards the end of the tasks T2 and T3 (indicating an increase in problem-related communication), while such trends are not as evident in the case of experts (task T2), or not present at all (task T3).

Finally, the data presented in Figure 4 reveals the influence of experience on the distribution of FBS processes. While novices' data remains largely the same, the experts use previous experience to ground links from functions to behaviours, and from behaviours to structures. Consequently, experts are able to traverse function-behaviour and behaviour-structure links quicker and spend a smaller percentage of time communicating them. This accords with an increase in the percentage of time spent on Evaluation and Reformulation I.

\section{CONCLUSION}

This paper presents a study of the differences between inexperienced and experienced teams regarding their patterns in problem-solution space exploration. Particularly, the hypotheses that experts converge to the solution quicker and spend more time exploring the solution space were tested. Although the systemic patterns found follow the theoretically-based expectations, the analysis of the simulation experiments results revealed some limitations of the model as currently implemented. These limitations include its inability to capture differences between the number of Evaluation and Reformulation I processes, as well as the lack of capacity to represent Reformulation II, Reformulation III and Documentation processes. Further, the data collected by utilising the computational model includes examples of very short tasks solved in a couple of time steps (the minimal number of steps needed to solve a task was three). In practice, the participants would most likely try to improve the solution even though it satisfies all of the requirements. Additionally, the inconsistency with the empirical finding that experts propose fewer solutions than novices, is likely a consequence of a lack of granularity of the agents' grading system where many structures are treated as equally good. To minimise these shortcomings, additional grading mechanisms will have to be implemented in the model. Further, motivational and affective mechanisms will be assigned to agents to drive them to improve and optimize solutions.

Despite these limitations, the experiments demonstrated the impact of learning and mental model change on the behaviour of agents, generating trends that accord with theory-based expectations. This cognitively-based agent model has shown its capacity to model team behaviour and has the capability of being used to test other hypotheses. It could be used to study the effect of churn on team behaviour, the 
impact of team structure on performance, the formation of de facto sub-teams, and the effect of design space generation, which can be used as a proxy for design creativity. The system can be used to produce the results of a very large set of simulations, the results of which could form the basis of a big data learning activity to uncover systemic behaviours that are not currently being measured.

\section{REFERENCES}

Ahmed, S., Wallace, K.M. and Blessing, L.T.M. (2003), "Understanding the differences between how novice and experienced designers approach design tasks", Research in Engineering Design, Vol. 14 No. 1, pp. 1-11. https://doi.org/10.1007/s00163-002-0023-z

Björklund, T.A. (2013), "Initial mental representations of design problems: Differences between experts and novices”, Design Studies, Vol. 34 No. 2, pp. 135-160. https://doi.org/10.1016/j.destud.2012.08.005

Cross, N. (2001), "Design cognition: results from protocol and other empirical studies of design activity", In: Newstatter, W. and McCracken, M. (Eds.), Design Knowing and Learning: Cognition in Design Education, Elsevier, Oxford, UK. pp. 79-103.

Cross, N. (2004), "Expertise in design: an overview”, Design Studies, Vol. 25 No. 5, pp. 427-441. https://doi.org/10.1016/j.destud.2004.06.002

Gero, J. S. (1990), "Design prototypes: A knowledge representation schema for design”, AI Magazine, Vol. 11 No. 4, pp. 26-36.

Gero, J.S., Jiang, H. and Williams, C.B. (2013), "Design cognition differences when using unstructured, partially structured, and structured concept generation creativity techniques", International Journal of Design Creativity and Innovation, Vol. 1 No. 4, pp. 196-214. https://doi.org/10.1080/21650349.2013.801760

Gero, J.S. and Kannengiesser, U. (2004), "The situated function-behavior-structure framework", Design Studies, Vol. 25 No. 4, pp. 373-391. https://doi.org/10.1016/j.destud.2003.10.010

Kahneman, D. (2011), Thinking, Fast and Slow, Farrar, Straus and Giroux, New York.

Kavakli, M. and Gero, J. (2002), "The structure of concurrent cognitive actions: a case study on novice and expert designers”, Design Studies, Vol. 23 No. 1, pp. 25-40. https://doi.org/10.1016/S0142-694X(01)00021-7

Liikkanen, L.A. and Perttula, M. (2009), "Exploring problem decomposition in conceptual design among novice designers", Design Studies, Vol. 30 No. 1, pp. 38-59. http://doi.org/10.1016/j.destud.2008.07.003

Lloyd, P. and Scott, P. (1997), "Discovering the design problem”, Design Studies, Vol. 15 No. 2, pp. $125-140$. https://doi.org/10.1016/0142-694X(94)90020-5

Maher, M.L. and Gero, J.S. (2002), “Agent models of 3D virtual worlds”, Proceedings of the 2002 Annual Conference of the Association for Computer-Aided Design in Architecture, Pomona, California, October 24-27, 2002, Association for Computer-Aided Design in Architecture, Pomona, pp. 127-138.

McComb, C., Cagan, J. and Kotovsky, K. (2015), "Rolling with the punches: An examination of team performance in a design task subject to drastic changes", Design Studies, Vol. 36, pp. 99-121. https://doi.org/10.1016/j.destud.2014.10.001

Milovanovic, J. and Gero, J.S. (2018), "Exploration of cognitive design behavior during design critiques", Proceedings of the 15th International Design Conference (DESIGN 2018), Dubrovnik, Croatia, May 21-24, 2018, The Design Society, Glasgow, pp. 2099-2110. https://doi.org/10.21278/idc.2018.0547

Perišić, M.M., Štorga, M. and Gero, J.S. (2017), "Building a computational laboratory for the study of team behaviour in product development", Proceedings of the 21st International Conference on Engineering Design (ICED 17), Vancouver, Canada, August 21-25, 2017, The Design Society, Glasgow, pp. 189-198.

Perišić, M.M., Štorga, M. and Gero, J.S. (2019), "Exploring the Effect of Experience on Team Behavior: A Computational Approach”, In: Gero, J.S. (Ed.), Design Computing and Cognition'18 (DCC'18), Springer, Cham, pp. 595-612. https://doi.org/10.1007/978-3-030-05363-5_32

Petre, M. (2004), "How expert engineering teams use disciplines of innovation", Design Studies, Vol. 25 No. 5 , pp. 477-493. https://doi.org/10.1016/j.destud.2004.05.003

Sun, G., Yao, S. and Carretero, J.A. (2016), “An experimental approach to understanding design problem structuring strategies”, Journal of Design Research, Vol. 14 No. 1, pp. 94-117. https://doi.org/10.1504/JDR.2016.074778

\section{ACKNOWLEDGMENTS}

This work is funded by Ministry of Science, Education and Sports of the Republic of Croatia, and Croatian Science Foundation project IP-2018-01-7269: Team Adaptability for Innovation-Oriented Product Development - TAIDE (http://www.taide.org), and has been supported in part by the National Science Foundation under grant numbers CMMI-1400466 and CMMI-1762415 to John Gero. Any opinions, findings, and conclusions or recommendations expressed in this material are those of the authors and do not necessarily reflect the views of National Science Foundation. 